\title{
Smart Material Robotic Technologies For Assisting Individuals with Upper Extremity Motor Impairment
}

\author{
Carlo Menon \\ Simon Fraser University \\ 8888 University Drive, Burnaby, V5A1S6, British Columbia, Canada \\ carlo_menon@sfu.ca
}

\section{Extended Abstract}

I envision a unified network of intelligent and mind-controlled robotic technologies for assisting individuals with motor impairments that are portable, wearable, minimal in size and affordable. They will:

- Continuously monitor health conditions;

- Assess recovery progress;

- Autonomously administer high-dose rehabilitation interventions to groups of patients in their homes; and

- Assist with independent living.

Currently, my research program primarily focuses on upper extremities (UE). I propose the following objectives:

1. Technology — Develop an innovative, UE close-fitting assistive sleeve based on transformative smart materials and robotics that detects bio-signals, and accordingly, promotes UE movements.

2. Interventions - Validate novel rehabilitation interventions enabled by the new technology to facilitate UE motor recovery in individuals with stroke.

3. Mechanisms - Monitor their brain reorganization and unveil mechanisms underlying recovery of motor function.

4. Assistance - Provide evidence that the robotic sleeve enhances independence.

5. Translation - Determine research priorities and implement integrated knowledge translation through direct involvement of stakeholders in all phases of the research.

By advancing knowledge in neurorehabilitation, and validating novel rehabilitation strategies, significant steps will be made to improve the health and quality of life of individuals living with stroke, which represents over 62 million people worldwide.[1] Furthermore, by facilitating recovery and enabling independent living, the long-term benefit of this research will be a reduced economic burden for both the people in need of assistance and the healthcare system.

\section{References}

[1] K. Strong, C. Mathers, and R. Bonita, "Preventing stroke: saving lives around the world," Lancet Neurol., vol. 6, no. February, pp. 182-187, 2007. 